\title{
Evaluación de la respuesta de la comunidad vegetal post-incendio en el MUP N'82 "Sierra de los Donceles" (2084 ha) de Hellín (Albacete) apoyado en el tratamiento de productos geomáticos públicos: escenas de satélite y LiDAR
}

Evaluation of the response of the post-fire plant community in the MUP N82 "Sierra de los Donceles" (2084 ha) of Hellín (Albacete) supported in the treatment of public geomatics products: satellite scenes and LiDAR

\author{
Peña, E.
}




\section{Resumen}

El fuego es probablemente el fenómeno destructivo más importante que afecta a las comunidades vegetales naturales o seminaturales, teniendo especial importancia en áreas mediterráneas. El 1 de julio de 2012 se produjo un incendio en la Sierra de los Donceles, en el sureste de la provincia de Albacete (Castilla-La Mancha, España), cuyo perímetro afectó a más de 7000 ha en las provincias de Albacete y Murcia. Tras pasar varios años después de dicho evento, se pretende con este trabajo evaluar los daños producidos a la vegetación en la zona afectada a partir del análisis de la escena de satélite pre y post-incendio y de la nube de puntos Light Detection and Ranging (LiDAR) debidamente procesada. Los datos obtenidos por esta metodología serán contrastados con un trabajo de campo en la zona no incendiada de dicho monte. El desarrollo de metodologías y procedimientos para aplicar éstas nuevas tecnologías puede ser una buena herramienta de trabajo a tener en cuenta como opción para el futuro de la profesión.

Palabras clave: Fuego, gestión forestal, SIG, teledetección.

\section{Summary}

Fire is probably the most important destructive phenomenon that affects natural or semi-natural plant communities, having special importance in Mediterranean areas. On July 1, 2012 there was a fire in the Sierra de los Donceles, in the southeastern of the province of Albacete (Castilla-La Mancha, Spain), whose perimeter affected more than 7000 ha in the provinces of Albacete and Murcia. After several years had passed since the event, the aim of this work is to evaluate the damage to the vegetation in the affected area from the analysis of the pre and post-fire satellite scene and the Light Detection and Ranging (LiDAR) point cloud properly processed. The data obtained by this methodology will be contrasted with a field work in the unburned zone of said mountain area. The development of methodologies and procedures to apply these new technologies can be a good work tool to consider as an option for the future of the profession..

Keywords: fire, forest management, GIS, remote sensing. 


\section{Introducción}

La reciente posibilidad de acceder a información geomática pública, escenas de misiones espaciales y productos LiDAR (acrónimo del inglés, Light Detection and Ranging o Laser Imaging Detection and Ranging) en el contexto de este trabajo, permite abordar procesos de análisis de mucha utilidad en diferentes aplicaciones en el ejercicio de la profesión de ingeniería forestal, y en particular en el caso de la evaluación de consecuencias de incendios forestales. El empleo de Sistemas de Información Geográfica (SIG) facilita la digitalización de todo tipo de información referida al terreno, a herramientas algo más novedosas como el empleo de la tecnología LiDAR para obtener parámetros dasométricos de forma continua en grandes superficies a bajo coste, mejorando las técnicas convencionales como el inventario clásico por conteo pie a pie.

Las estadísticas que se elaboran sobre los daños producidos por los incendios forestales no suelen seguir una metodología normalizada y están basados, en la mayoría de los casos, en estimaciones realizadas a pie de campo (Navarro et al., 2001). Dado el alto coste de los inventarios de campo el empleo de la teledetección en los incendios forestales está más que extendido y su utilidad está claramente justificada. Analizando las situaciones pre y post-incendio se pueden extraer conclusiones del estado de la vegetación a través de numerosos índices: Normalized Difference Vegetation Index ó Índice de Vegetación Diferencial Normalizado (NDVI), Difference Normalized Burn Ratio ó índice de quemado (dNBR), etc.

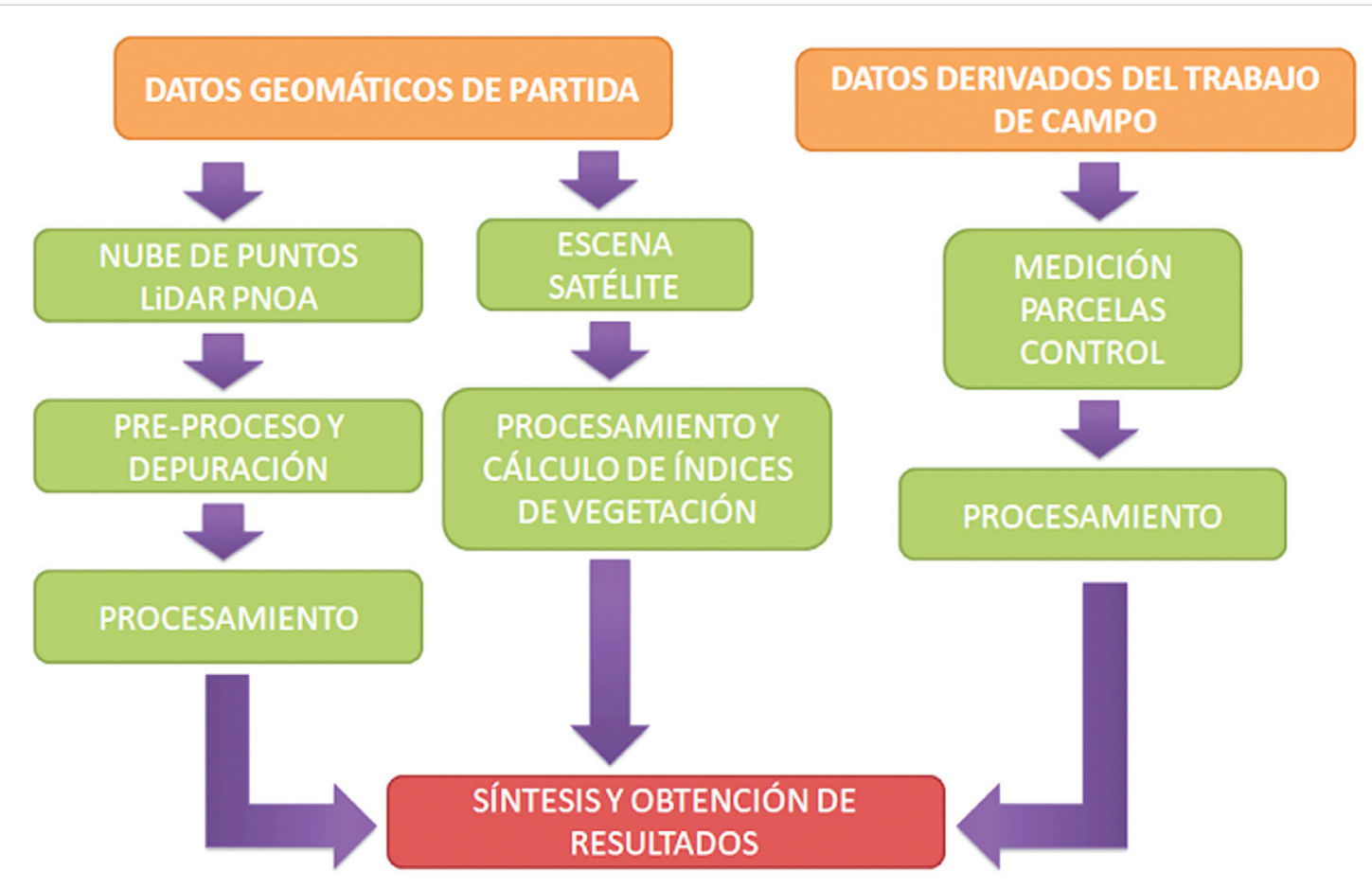

Figura 1. Esquema del flujo de trabajo desarrollado en el presente trabajo. 
En cuanto al uso de la tecnología LiDAR, combinada con la información obtenida por teledetección y el oportuno trabajo de campo, es posible cuantificar y evaluar el estado forestal de la vegetación (alturas, coberturas, etc.) a partir del correcto tratamiento y procesado de la nube de puntos. El uso de esta tecnología debido a su gran versatibilidad y precisión en los resultados obtenidos está cada vez más extendida (Díaz et al., 2007; González et al., 2016; Jiménez et al., 2017).

\section{Material y métodos}

El flujo de trabajo llevado a cabo en el desarrollo de la metodología para evaluar y cuantificar las pérdidas de biomasa ocasionadas por el incendio en la Sierra de los Donceles (MUP N82) mediante teledetección y LiDAR se puede observar a continuación (ver Fig. 1 en página anterior):

El procedimiento a seguir consta de tres partes diferenciadas: una medición de parcelas control en campo, un análisis y procesamiento de la nube de puntos LiDAR y el procesamiento y cálculo de índices de vegetación a partir de la escena satélite.

\section{1. Área de estudio y objetivos}

Las coordenadas según el sistema de coordenadas universal transversal de Mercator (en inglés Universal Transverse Mercator, UTM) entre las que se encuentra la zona objeto de estudio son (Fig.2):

$$
\text { X: } 607600-619100 \quad Y: 4249000-4255000
$$

El incendio del 1 de julio de 2012 se propagó a gran velocidad debido a las condiciones climáticas y al alto contenido en combustible, ya que existía un sotobosque denso formado principalmente por especies de alta inflamabilidad. Dicho incendio afectó casi a la totalidad de este monte (2084 ha), salvándose aproximadamente unas 530 ha.

El objetivo general perseguido con este trabajo es evaluar y cuantificar los daños producidos por el incendio ocurrido el 1 de julio de 2012 en la zona objeto de estudio (Sierra de los Donceles, Hellín). Los objetivos parciales son:

- Valoración del estado de evolución de la vegetación según biomasa existente en la situación pre y post-incendio.

- Muestreo de parcelas en campo para contrastar y validar los resultados obtenidos.

— Obtención de diferentes índices de vegetación (NDVI, dNBR...) en situaciones distintas (pre-incendio y post-incendio) a partir de la escena de satélite, para valorar la evolución del cambio de la vegetación tras el incendio. 


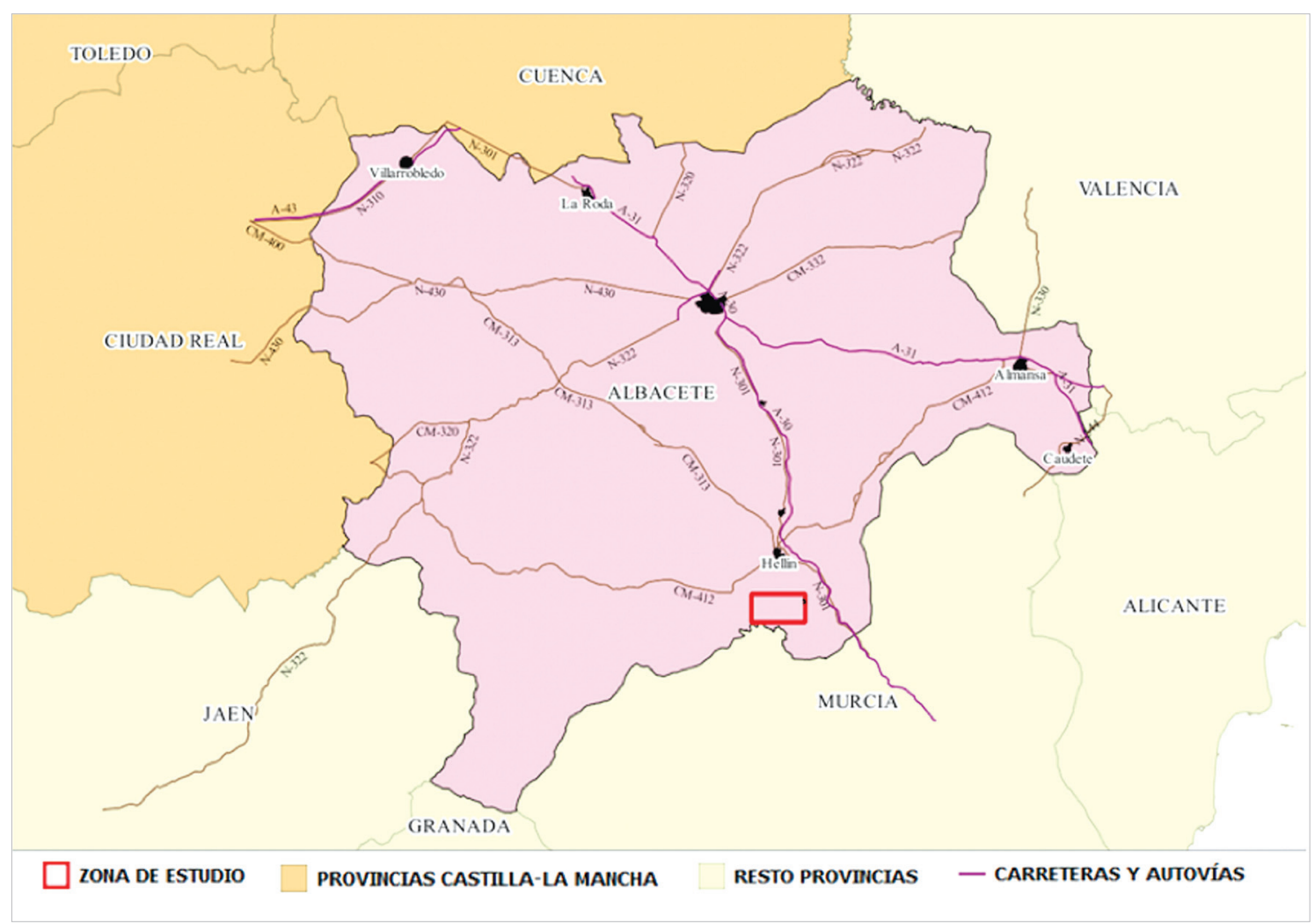

Figura 2. Localización de la zona de estudio enmarcada en rojo (sistema de referencia ETRS89, EPSG: 25830).

- Obtención de la nube de puntos LiDAR PNOA con la información espacial de la zona de estudio para calcular: el modelo de alturas de vegetación (CHM) y fracción de cabida cubierta en la situación pre-incendio.

\subsection{Obtención datos de partida}

En la realización del presente proyecto se han utilizado diferentes tipos de datos geomáticos exclusivamente públicos para el total desarrollo del mismo. Así, tenemos las siguientes fuentes de datos:

- Nube LiDAR PNOA 2009 de baja densidad (0.5 puntos/m2) obtenida del Centro Nacional de Información Geográfica (CNIG).

- Escenas de satélite de la misión espacial LANDSAT-7 de la zona objeto de estudio con la siguiente serie temporal: dos meses antes del incendio y dos meses después del incendio obtenido de U.S. Geological Survey (USGS).

- Medición de parcelas control (30x30 m) en la zona no incendiada de la "Sierra de los Donceles" para contrastar los resultados obtenidos mediante teledetección y LiDAR. 


\subsection{Procesado de los datos}

\subsubsection{Tratamiento de la nube de puntos LiDAR PNOA 2009}

La nube de puntos bruta descargada del CNIG tendrá que pasar por varios procesos: una transformación de formato (de ficheros con extensión *.LAZ convertidos a *.LAS); varias depuraciones para reclasificar puntos y eliminar outliers (puntos atípicos) siendo errores típicos que puntos de vegetación aparezcan como edificación, o viceversa, o que aparezcan clasificados erróneamente puntos que son efecto ruido, tales como pájaros, torretas, cableado, etc; por último, con los resultados obtenidos del procesado de la nube de puntos LiDAR y mediante el software estadístico específico STATGRAPHICS se ajustará un modelo para el cálculo de la biomasa pre-incendio del MUP No82 (al disponer de datos reales de la zona) y se extrapolarán a la superficie total del incendio.
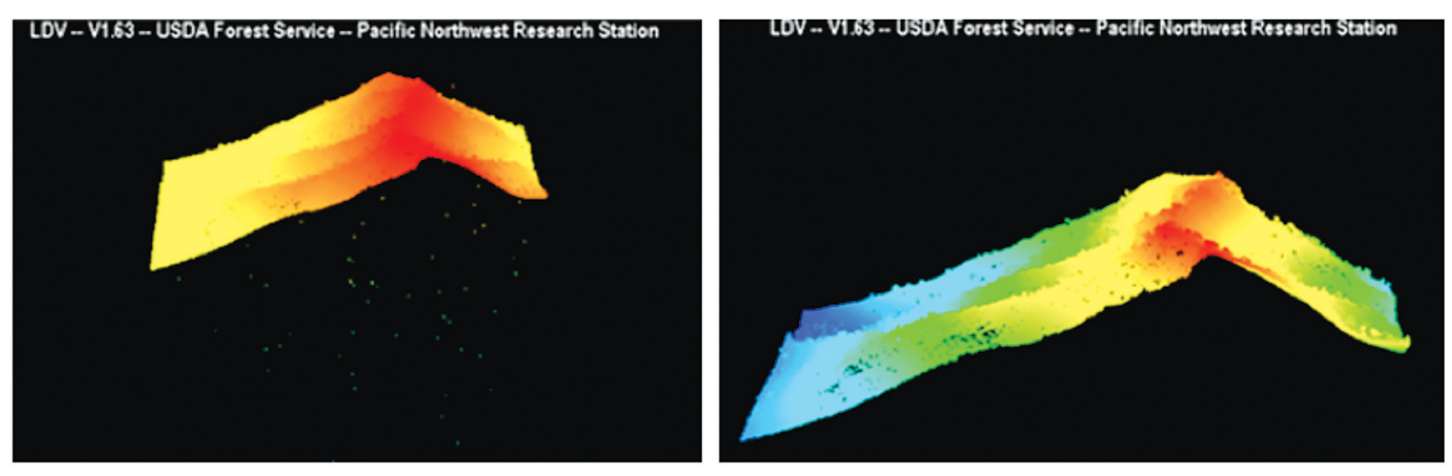

Figura 3. Detalle de la nube de puntos LiDAR bruta “sin filtro" y "con filtro'.' (Fuente: FUSION).

\subsubsection{Tratamiento de la escena de satélite.}

A partir de la escena satélite disponible se calcularán diversos índices de vegetación. Los índices de vegetación son combinaciones de las bandas espectrales registradas por los satélites de Teledetección, cuya función es obtener parámetros no afectados de las condiciones particulares de la observación para realzar la vegetación, en cierto estado, en función de su respuesta espectral. Para este estudio nos centraremos en obtener los más empleados en estas aplicaciones:

dNBR (Difference Normalized Burn Ratio): es el resultado de normalizar la diferencia de reflectancias entre la banda del infrarrojo cercano (NIR, banda 4) y una del infrarrojo medio (SWIR, banda 7) aplicando la siguientes fórmulas (Keeley, 2009):

$$
\begin{aligned}
\mathrm{NBR} & =\frac{(\mathrm{NIR}-\mathrm{SWIR})}{(\mathrm{NIR}+\mathrm{SWIR})} \\
\mathrm{dNBR} & =\mathrm{NBR}_{\text {pre--incendio }}-\mathrm{NBR}_{\text {post-incendio }}
\end{aligned}
$$


NDVI (Normalized Difference Vegetation Index ó Índice de Vegetación Diferencial Normalizado): es un índice de vegetación que se utiliza para estimar la cantidad, calidad y desarrollo de la vegetación con base a la intensidad de la radiación de ciertas bandas del espectro electromagnético que la vegetación fotosintéticamente activa emite o refleja. Para su cálculo es necesaria la información que se encuentra en las bandas roja e infrarroja mediante la aplicación de la siguiente fórmula (Rouse et al., 1974):

$$
\mathrm{NDVI}=\frac{(\mathrm{NIR}-\mathrm{RED})}{(\mathrm{NIR}+\mathrm{RED})}
$$

El cálculo de los diversos índices será llevado a cabo con la calculadora ráster de QGIS 2.18 (un Sistema de Información Geográfica de código abierto licenciado bajo GNU - General Public License). Tras realizar el cálculo del índice NDVI junto con la información obtenida de la biomasa en las parcelas medidas mediante relación alométrica para biomasa (Ruiz-Peinado, 2011) se ajustará un modelo BIOMASA-NDVI con el que se valorará la variación que ha sufrido la vegetación durante el periodo de estudio (situación pre y post-incendio).

2.3.3. Medición parcelas y cálculo parámetros forestales de interés.

En dichas parcelas se han medido los siguientes parámetros forestales:

— Diámetro normal $(\mathrm{cm})$ a la altura de 1,30 $\mathrm{m}$. Se harán dos mediciones en cruz y se obtendrá un diámetro medio a partir de las dos mediciones.

- Altura (m). Se hará una única medición mediante hipsómetro Suunto.

— Diámetro de copa (m). Se harán dos mediciones en cruz y se calculará un diámetro de copa medio entre las dos mediciones realizadas.

A partir de esta información, al tener datos reales de campo, podremos validar y ajustar modelos de regresión para el cálculo de la biomasa utilizando dichos datos de campo y la información obtenida a partir del tratamiento de las escenas de satélite y de la nube de puntos LiDAR PNOA.

\section{Resultados y Discusión}

El resultado de los procesamientos anteriores permite obtener las pérdidas de biomasa ocasionadas por el incendio. Este proceso lo abordaremos desde dos perspectivas:

- Mediante obtención de un modelo BIOMASA-NDVI a partir de los datos de las parcelas (relación alométrica para la biomasa de Ruiz-Peinado) y del índice de vegetación NDVI. 
- Mediante la obtención de un modelo para la biomasa LiDAR a partir de la relación alométrica para la biomasa de Ruiz-Peinado y las métricas LiDAR de cada una de las parcelas.

El proceso de obtención de los modelos se realizará mediante el software STATGRAPHICS (Tab.1).

Tabla 1. Cuadro resumen de las relaciones alométricas obtenidas con la metodología aplicada.

\begin{tabular}{ccccc}
\hline Información & Modelo & $\begin{array}{c}\text { Resultado } \\
\left(\mathrm{kg} \mathrm{ha}^{-1}\right)\end{array}$ & $\begin{array}{c}\text { Eabs } \\
\left(\mathrm{kg} \mathrm{ha}^{-1}\right)\end{array}$ & $\begin{array}{c}\text { Erel } \\
(\%)\end{array}$ \\
\hline Biomasa Ruiz-Peinado & $\mathrm{W}=0.039 \mathrm{x} \mathrm{d}^{2} \times \mathrm{h}$ & 3848,13 & 1612,51 & 41,90 \\
\hline Biomasa-NDVI & $\mathrm{W}=36209 \mathrm{x} \mathrm{NDVI}^{2.9975}$ & 3568,54 & 1058,90 & 29,67 \\
\hline Biomasa-LiDAR & $\begin{array}{c}\mathrm{W}=225,896+ \\
312,713 * \text { Elev_kurtosis }\end{array}$ & 3711,62 & 1173,86 & 31,63 \\
\hline
\end{tabular}

En las Fig. 4, 5, 6 y 7 se puede observar la representación de los resultados obtenidos así como ver el cambio brusco en la cantidad de biomasa antes y después del incendio.

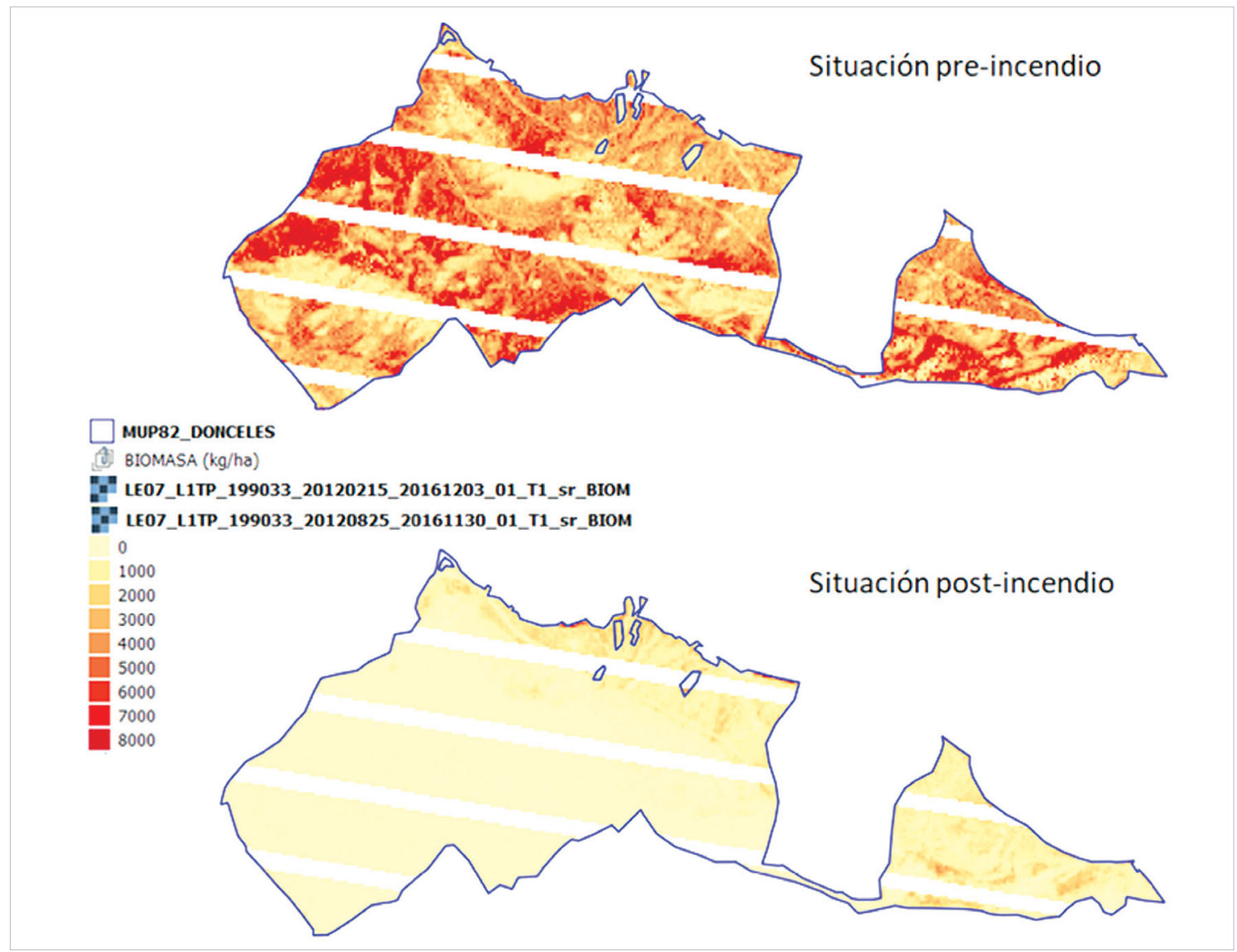

Figura 4. Visualización biomasa $\left(\mathrm{kg} \mathrm{ha}^{-1}\right)$ antes y después del incendio en el MUP Nº8. 


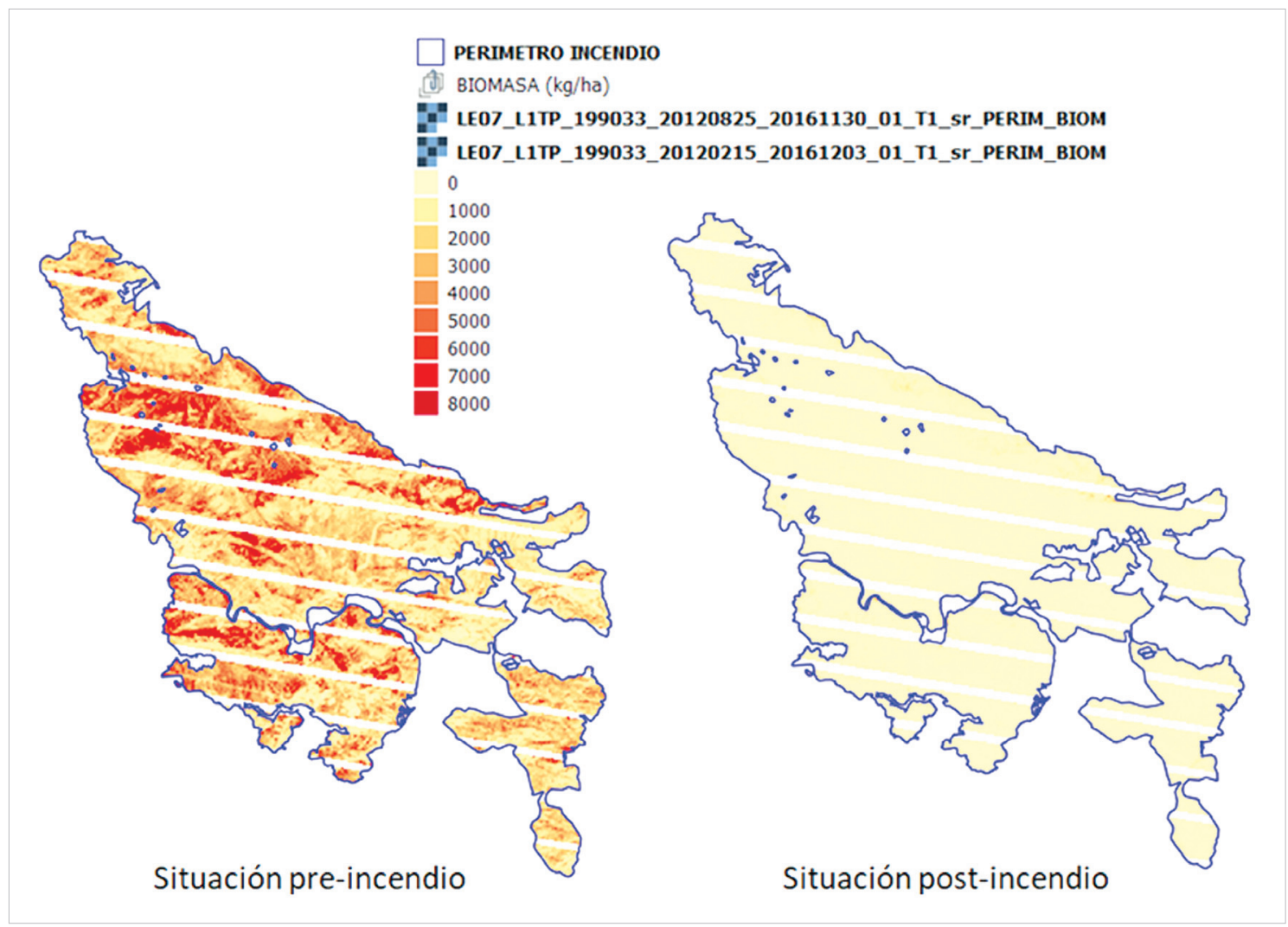

Figura 5. Cálculo pérdida biomasa $\left(\mathrm{kg} \mathrm{ha}^{-1}\right)$ para la superficie completa del incendio.

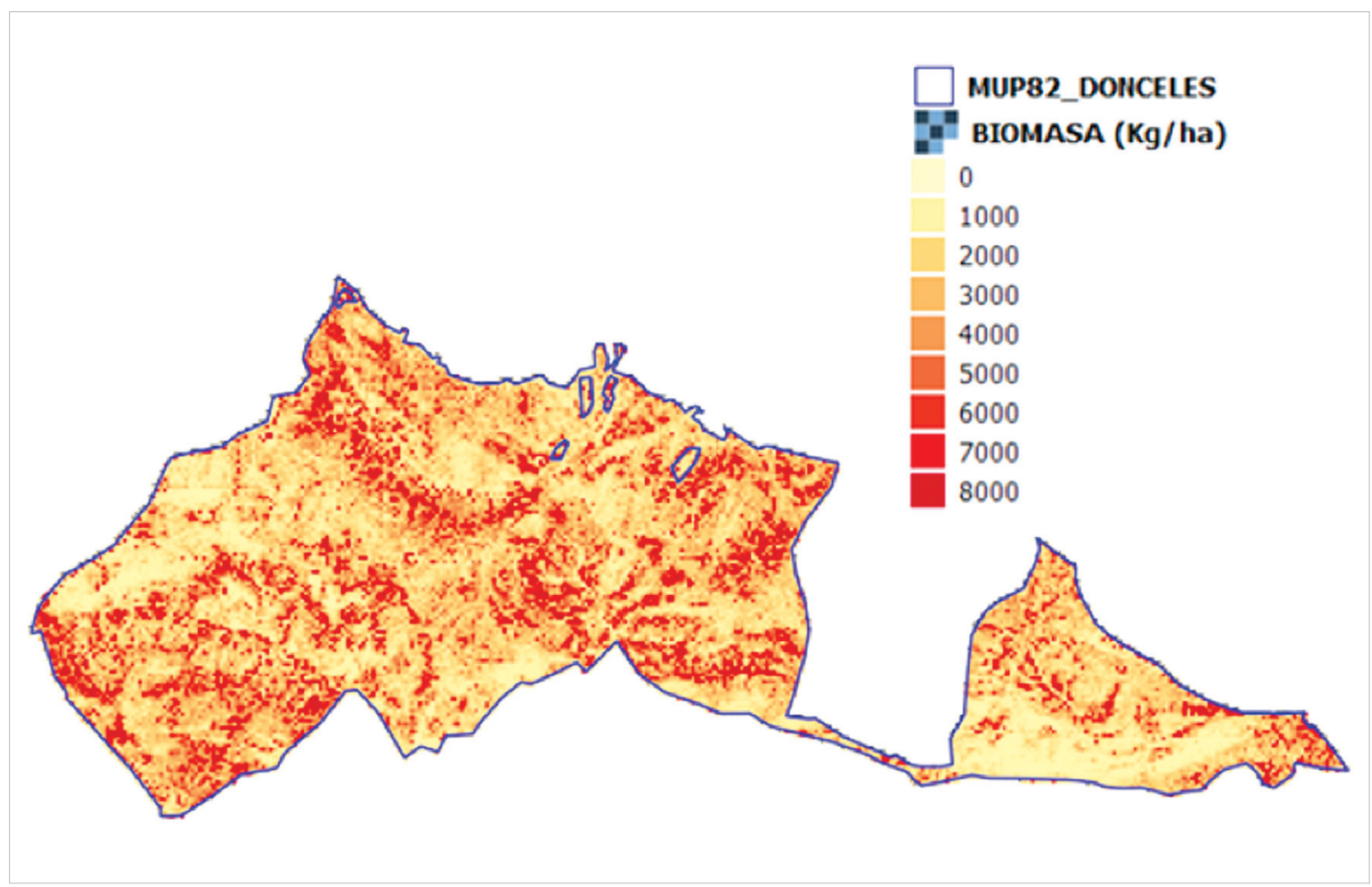

Figura 6. Visualización biomasa $\left(\mathrm{kg} \mathrm{ha}^{-1}\right)$ antes del incendio en el MUP No 82 . 


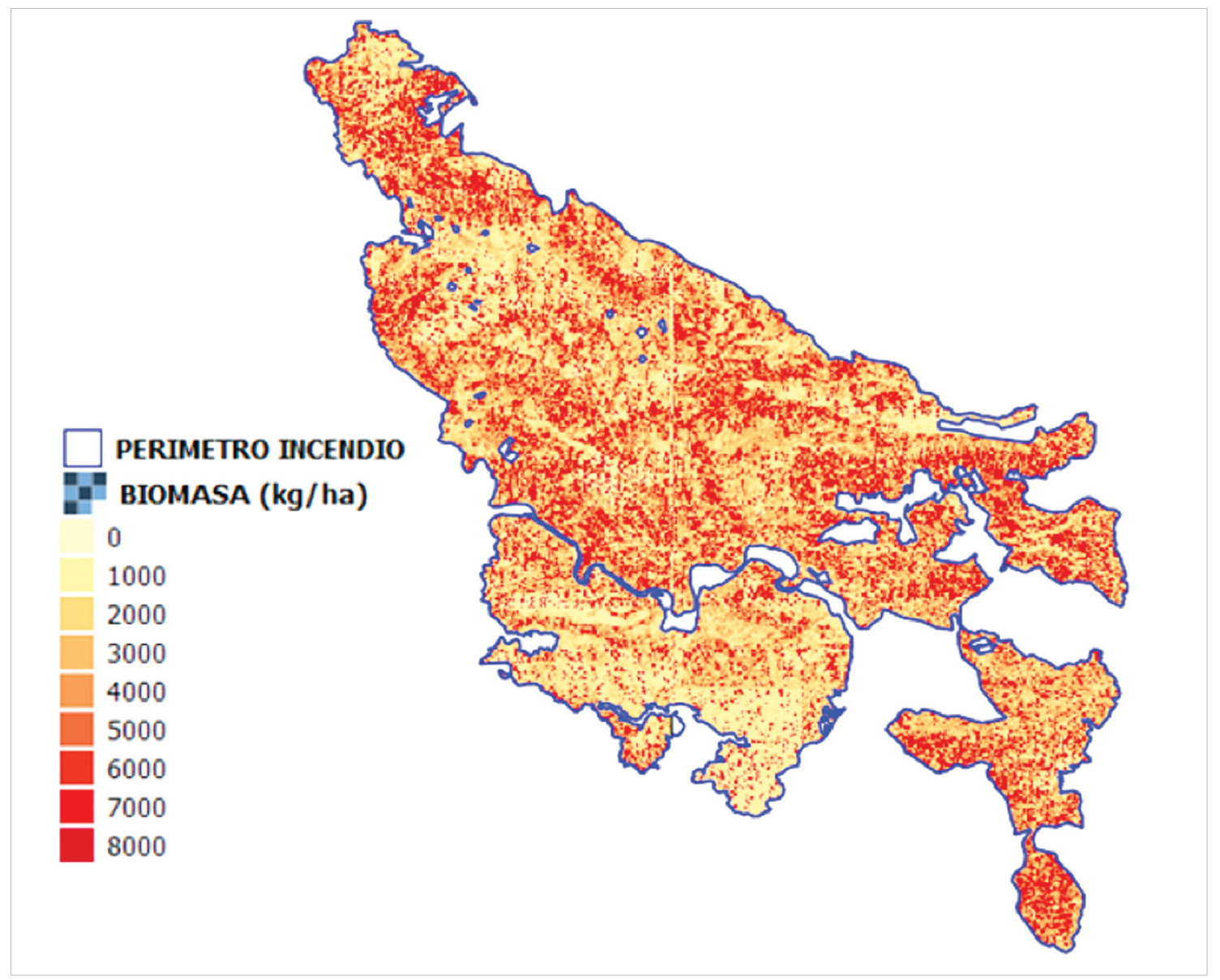

Figura 7. Visualización biomasa $\left(\mathrm{kg} \mathrm{ha}^{-1}\right)$ antes del incendio para el perímetro completo del incendio.

Los resultados obtenidos con el desarrollo de esta metodología han sido satisfactorios, debido a la utilidad, potenciabilidad y versatibilidad de los datos empleados en el desarrollo del mismo. A la vista de los resultados obtenidos, se han cumplido los objetivos perseguidos en el presente proyecto:

- Los índices de vegetación (dNBR y NDVI) han cumplido el objetivo de valorar la evolución del cambio de la vegetación tras el incendio. Estos índices han sido de gran utilidad. El primero ha permitido delimitar la superficie afectada por el incendio (para extrapolar los resultados a toda la superficie) $y$ el segundo nos ha permitido obtener un modelo para la biomasa, esencial para conocer las pérdidas ocasionadas por dicho evento. La ventaja del uso de sistemas remotos es la cantidad de información que se puede extraer de su correcto procesado y no visible al ojo humano.

- Los datos derivados de la nube de puntos LiDAR han cumplido el objetivo de caracterizar el estado de la masa pre-incendio (alturas de vegetación, fracción de cabida cubierta y biomasa). Además, a partir de la obtención de las 
métricas LiDAR por parcela ha sido posible ajustar un modelo para la biomasa existente pre-incendio y extrapolarlos al total de la superficie.

- Los datos de las parcelas control inventariadas han cumplido el objetivo de contrastar y validar los resultados obtenidos mediante teledetección y LiDAR. El trabajo de campo ha sido indispensable para validar y obtener los resultados finales. Gracias a los datos recogidos de cada parcela ha sido posible obtener la biomasa por parcela (según relación Ruiz-Peinado), para despúes poderla relacionar con el índice de vegetación NDVI y obtener las pérdidas de biomasa ocasionadas por el incendio para el MUP No82 y, al extrapolar los resultados, para toda la superficie afectada por el mismo.

En este trabajo no consta (debido a falta de información LiDAR tras el incendio), pero de cara a futuros trabajos sería interesante disponer de información LiDAR disponible en varias fechas, para poder verificar los cambios que se producen (mediante la comparación de los resultados inicial y final). No obstante, el uso de datos LiDAR (aún siendo de baja densidad de puntos) aporta gran volumen de información y resultados fiables, apoyado siempre con un trabajo de campo para contrastar y validar dichos resultados.

\section{Conclusión}

El desarrollo de metodologías basadas en datos geomáticos públicos (escenas de satélite y LiDAR) y apoyados y contrastados por trabajos de campo es de gran utilidad para analizar cambios y variaciones en los ecosistemas naturales. La información obtenida tras los distintos procesamientos ofrece gran utilidad al técnico ó gestor forestal para la compresión del espacio de trabajo íntegra (incluso en zonas poco accesibles) y espacialmente, de forma continua abarcando toda la extensión de estudio. Además, permite que los resultados obtenidos puedan ser actualizados con facilidad y rapidez, pudiendo mostrar las variaciones que se producen en el medio natural y elegir el modo de actuar en la zona frente a los resultados obtenidos (proponer zonas que requieran tratamientos de suelo para favorecer regeneración, delimitar zonas de alta severidad de incendio, etc.).

\section{Bibliografía}

CNIG. "Centro Nacional de Información Geográfica". Recuperado de: http://centrodedescargas.cnig.es/CentroDescargas/index.jsp

Díaz F.R., Acosta M.M., Carrillo F.A., Buendía E.R., Flores E.A. y Etcheveres-Barra J.D. (2007): Determinación de ecuaciones alométricas para estimar biomasa y carbono en Pinus Patula Schl. et Cham. Madera y Bosques. 13 (1): 25-34. https://doi.org/10.21829/ myb.2007.1311233 
González S., De las Heras J. y Moya D. (2016) “Resilience of Mediterranean terrestrial ecosystems and fire severity in semiarid areas: Responses of Aleppo pine forests in the short, mid and long term". Universidad de Castilla-La Mancha.

Jiménez E., Vega J.A., Fernández-Alonso J.M., Vega-Nieva D., Ortiz L., López-Serrano P.M. y López-Sánchez C.A. (2017). "Estimation of aboveground forest biomass in Galicia (NW Spain) by the combined use of LiDAR, LANDSAT ETM+ and National Forest Inventory data". iForest 10: 590-596 [online 2017-05-15]. https://doi.org/10.3832/ifor $1989-010$

Keeley, J.E. (2009). Fire intensity, fire severity and burn severity: A brief review and suggested usage. International Journal of Wildland Fire, 18(1), 116-126. https://doi.org/ 10.1071/WF07049

Navarro R., Fernández P. y Escuin S. (2001). “Evaluación de daños producidos por incendios forestales mediante imágenes satélite. Propuesta de Restauración". Universidad de Córdoba.

Navazo G., Nieto A. y Moreno G. “Análisis de incendios forestales mediante sistemas de información geográfica y teledetección. Estudio de caso en Sierra de Gata (2015) ”. Universidad Extremadura.

Rouse J.W., Haas R.H., Deering D.W. y Sehell J.A. (1974): Monitoring the vernal advancement and retrogradation (Green wave effect) of natural vegetation. Final Rep. RSC 19784, Remote Sensing Center, Texas A\&M Univ., College Station.

Ruiz-Peinado R., Del Rio M. y Montero G. (2011) "New models for estimating the carbon sink capacity of Spanish softwood species". Instituto Nacional de Investigación y Tecnología Agraria y Alimentaria (INIA)

USGS. "Landsat Spectral Indices Product Guide” (versión 3.5, 2017). Recuperado de: https:// landsat.usgs.gov/sites/default/files/documents/si_product_guide.pdf 